\title{
Generation and Characterization of Intense Ultralow-Emittance Electron Beams for Compact X-Ray Free-Electron Lasers
}

\author{
E. Prat $\odot,{ }^{1, *}$ P. Dijkstal® ${ }^{1,2}$ M. Aiba, ${ }^{1}$ S. Bettoni $\odot,{ }^{1}$ P. Craievich $\odot,{ }^{1}$ E. Ferrari, ${ }^{1}$ R. Ischebeck $\odot,{ }^{1}$ F. Löhlø, ${ }^{1}$ \\ A. Malyzhenkov, ${ }^{1}$ G. L. Orlandi® ${ }^{1}{ }^{1}$ S. Reiche, ${ }^{1}$ and T. Schietinger ${ }^{1}$ \\ ${ }^{1}$ Paul Scherrer Institut, CH-5232 Villigen PSI, Switzerland \\ ${ }^{2}$ ETH Zürich, Department of Physics, CH-8093 Zürich, Switzerland
}

(Received 9 October 2019; published 6 December 2019)

\begin{abstract}
The transverse emittance of the electron beam is a fundamental parameter in linac-based $\mathrm{x}$-ray freeelectron lasers (FELs). We present results of emittance measurements carried out at SwissFEL, a compact x-ray FEL facility at the Paul Scherrer Institute in Switzerland, including a description of the novel highresolution measurement techniques and the optimization procedure. We obtained slice emittance values at the undulator entrance down to $200 \mathrm{~nm}$ for an electron beam with a charge of $200 \mathrm{pC}$ and an rms duration of $30-40$ fs. Furthermore, we achieved slice emittances as low as $100 \mathrm{~nm}$ for $10 \mathrm{pC}$ beams with few fs duration. These values set new standards for electron linear accelerators. The quality, verification, and control of our electron beams allowed us to generate high-power FEL radiation for a wavelength as short as $0.1 \mathrm{~nm}$ using an electron beam with an energy of only $6 \mathrm{GeV}$. The emittance values demonstrated at SwissFEL would allow producing hard x-ray FEL pulses with even lower-energy beams, thus paving the way for even more compact and cost-effective FEL facilities.
\end{abstract}

DOI: 10.1103/PhysRevLett.123.234801

X-ray free-electron lasers (FELs) driven by linacs are cutting-edge research instruments to investigate matter with temporal and spatial resolutions at the atomic level [1-7]. The FEL process requires extremely high-brightness electron beams: for $\mathrm{x}$-rays, the electron beam needs to have a peak current at the kA level, a relative energy spread between 0.01 and $0.1 \%$, emittances normalized to beam energy $\left(\varepsilon_{n}=\gamma \varepsilon\right.$ with $\gamma$ the Lorentz factor) at the micrometer level or below, and transverse beam sizes of a few tens of micrometers or smaller.

Of particular importance is the transverse emittance, which has a fundamental impact on FEL performance. Transversely coherent FEL radiation is produced if $\varepsilon_{n} / \gamma \approx$ $\lambda / 4 \pi$ [8], where $\lambda$ is the FEL radiation wavelength. This requirement implies that smaller emittances enable freeelectron lasing with lower-energy beams, which in turn are accessible by more compact and affordable accelerators. Moreover, for a given beam energy, the FEL performance is improved for smaller emittances: higher pulse energies are obtained in shorter undulator lines. The influence of the emittance on the FEL process is illustrated with an example in Fig. 1: a smaller emittance either means that a higher FEL pulse energy can be reached at the same electron beam energy, or, conversely, that the same FEL pulse energy can be reached with lower electron beam energy.

The distance over which the electrons interact with each other in the FEL process, i.e., the FEL cooperation length, is typically a small fraction of the total electron pulse duration. Therefore, for FEL facilities it is fundamental to control the local emittance along the electron bunch, commonly named slice emittance. Time-resolved diagnostics are extremely useful to measure not only the slice

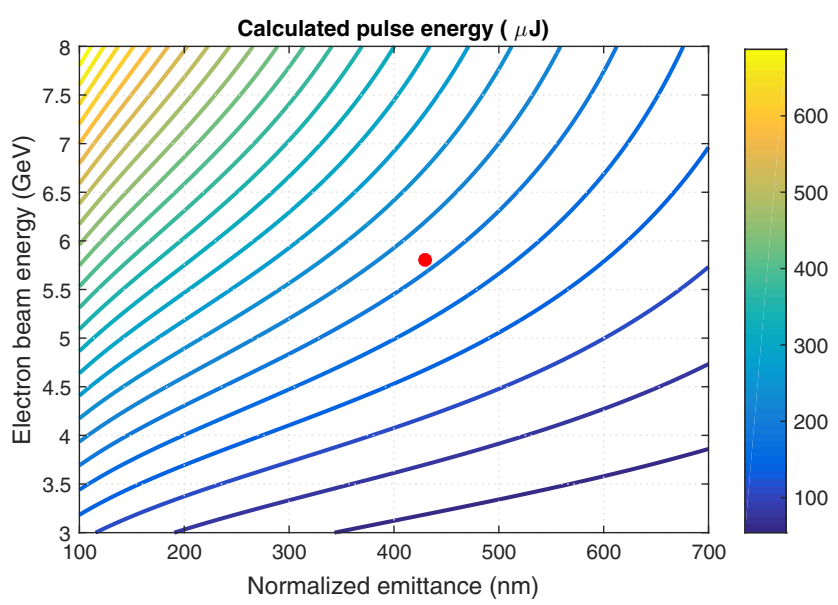

FIG. 1. Calculated FEL energy using the Ming Xie model [9] for a radiation wavelength $\lambda$ of $0.1 \mathrm{~nm}$ as a function of the electron beam energy and normalized emittance. The undulator parameter $K$ is 1.2 for all cases and the undulator period $\lambda_{u}$ is adjusted to fulfill the FEL resonant condition $\lambda=\lambda_{u}\left(1+K^{2} / 2\right) / 2 \gamma^{2}$. The other relevant beam parameters are constant: the peak current is $2 \mathrm{kA}$, the bunch charge is $100 \mathrm{pC}$, the average $\beta$ function in the undulator is $10 \mathrm{~m}$, and the energy spread is $1 \mathrm{MeV}$. The case corresponding to the SwissFEL conceptual design report (CDR) [10] (5.8 GeV, $430 \mathrm{~nm}, \lambda_{u}=15 \mathrm{~mm}$ ) is marked with a red dot. 
emittance but also the slice optics and trajectory. An optics mismatch or a misalignment along the electron bunch may deteriorate the FEL performance and induce a growth in the emittance of the whole electron beam, called projected emittance. The projected emittance is a general indicator of the transverse beam quality and thus needs to be measured and minimized.

Extensive research and development has gone into optimizing the emittance of electron beams at $\mathrm{x}$-ray FEL facilities [11-16]. Here we present projected and slice emittance measurements performed at SwissFEL, a compact hard $\mathrm{x}$-ray FEL facility that recently came into operation at the Paul Scherrer Institute in Switzerland [7,10,17].

Figure 2 shows a sketch of SwissFEL. The total length of the facility is about $700 \mathrm{~m}$. Electrons are generated in a radio frequency (rf) photoinjector [18] in bunches of $200 \mathrm{pC}$ charge (standard operation mode). For shorter FEL pulses, however, the beam charge is reduced down to $10 \mathrm{pC}$. The longitudinal laser profile has a Gaussian shape with rms durations of about 3 ps for $200 \mathrm{pC}$ and 1.4 ps for $10 \mathrm{pC}$. The transverse distribution has a truncated Gaussian shape, which according to our simulations results in better emittance than a full Gaussian distribution [19]. An $S$-band ( $3 \mathrm{GHz}$ ) booster accelerates the electron beam up to $300 \mathrm{MeV}$, and a $C$-band $(5.7 \mathrm{GHz}$ ) linac brings the electron beam to its final energy up to about $6 \mathrm{GeV}$. The electron beam is longitudinally compressed in two bunch compressors ( $\mathrm{BC} 1$ and $\mathrm{BC} 2)$. The electron beam generates FEL radiation in the undulator beam line with wavelengths between 0.1 and $0.7 \mathrm{~nm}$.

We present normalized emittance measurements for bunch charges of 200 and $10 \mathrm{pC}$, for uncompressed beam, after compression in $\mathrm{BC} 1$, and after nominal compression at the end of the linac. The slice emittance measurements are performed with transverse-deflecting structures (TDS) [20]: an $S$-band TDS with a temporal resolution of approximately $10 \mathrm{fs}$ after $\mathrm{BC} 1$, and two $C$-band TDS with a resolution of around $1 \mathrm{fs}$ at the end of the linac. The TDS streak the beam vertically to allow for time-resolved measurements in the horizontal plane, which is most critical due to the horizontally acting bunch compressors.

To optimize the final emittance we first minimize the emittance at the injector for uncompressed bunches, following the procedure explained in [15]. The electric field at the cathode is set to its maximum operational value of $100 \mathrm{MV} / \mathrm{m}$ to maximize the beam energy at the gun exit $(7.1 \mathrm{MeV})$ and to minimize the emittance contribution from rf fields in the gun. The laser transverse size at the cathode, the gun solenoid field, and the distance between the gun and the booster are chosen to counterbalance space-charge

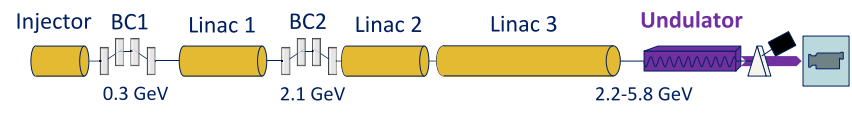

FIG. 2. SwissFEL schematic (not to scale). forces and the intrinsic (cathode) emittance for design current. These three parameters are set to an optimum found by simulations [21]. The laser size and the solenoid field are then empirically tuned to achieve the minimum emittance. The optimum laser rms beam size in both transverse planes is about $0.2 \mathrm{~mm}$ for $200 \mathrm{pC}$ and $0.08 \mathrm{~mm}$ for $10 \mathrm{pC}$. We also optimize other significant contributions that affect the source emittance: the laser is aligned with respect to the rf gun, the solenoid is aligned to the gun axis, the beam energy spread at the gun exit is minimized tuning the gun phase, the beam is aligned within the booster rf stations, and the transverse coupling is corrected with quadrupole magnets in the gun solenoid following the procedure described in [22].

Once the injector emittance is minimized, we aim at preserving it during beam acceleration, compression, and transport to the undulator entrance. Potential sources of emittance degradation include coherent synchrotron radiation (CSR) in the bunch compressors [23,24], transverse beam tilts caused by wakefields in the linacs or by compression, chromaticity, and dispersion effects. The optics are designed to have a small $\beta$ function at the last section of the bunch compressors, so that the transverse impact of the CSR kick is minimal. Moreover, the optics are smooth within the machine to keep chromatic effects under control. The firstand second-order transverse tilts are measured using slice diagnostics and corrected with quadrupoles and sextupoles magnets in the bunch compressors, as described in [25]. Figure 3 shows an example of transverse beam tilt correction applied at the second bunch compressor. Finally, the beam is centered at the different magnets within the lattice using standard beam-based alignment procedures. A random-walk algorithm used to maximize the FEL pulse energy sometimes helps to improve the final emittances. This is achieved by compensating the transverse beam tilts beyond the accuracy of our systematic measurement and correction approach.
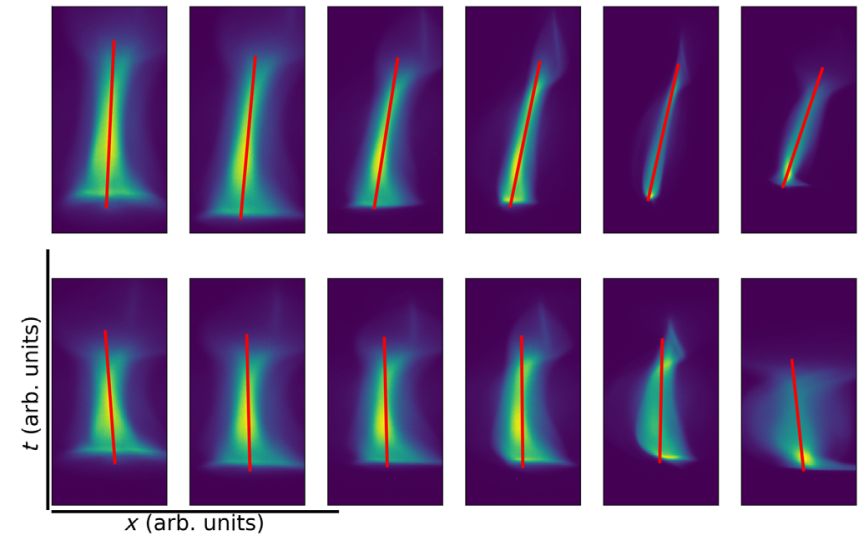

FIG. 3. Streaked beam images for different optics conditions before (top) and after (bottom) beam tilt correction. The red line indicates a linear fit to the beam image, whose slope is proportional to the tilt amplitude. 
We measure the emittances and optics of the beam at a certain location by measuring the beam sizes at a downstream position for different betatron phase advances between the measurement and the reconstruction points. We use quadrupole magnets to scan the phase advance in the measurement direction for a total range of up to $180^{\circ}$, to optimize the $\beta$ function for resolution purposes, and, in the case of the slice emittance measurements, to keep the phase advance in the streaking direction close to $90^{\circ}$ or $270^{\circ}$ so that the conversion from the TDS kick to the offset at the screen is maximized. The projected emittance is measured with the symmetric single-quadrupole scan technique described in [26]. Several quadrupole magnets are used for the slice emittance measurements, similar to the procedure in [15].

We employ wire scanners [27] and YAG screens [28] to measure the transverse beam sizes. The wire scanners have a higher resolution and are less invasive to beam operation, but they provide a multishot and 1D measurement that can only be used to obtain projected beam properties. The screens give single-shot and full 2D information so they are useful to retrieve not only the projected beam sizes but also the slice beam parameters and the transverse coupling. For the slice emittance measurements with the screens, we divide the streaked beam image into different bands corresponding to the temporal slices, with typically about 20 slices covering the full beam. Beam sizes are determined by applying Gaussian fits to the transverse beam profiles. Such fits appropriately represent the beam core contributing to the lasing process [15].

Measurement errors are evaluated by error propagation of the statistical beam-size uncertainties. We assume a minimum beam size error of 5\% and add shot-to-shot measurement variations in quadrature. For the slice emittance, we average the results over the five central slices. In this case, the reported errors reflect both the variation of the emittance along the slices and the statistical errors in the emittance measurement.

Considerable effort has gone into understanding and overcoming the emittance error arising from the profile monitor resolution, which is the most important systematic uncertainty in the emittance determination. Other systematic errors, including screen calibration and beam energy and quadrupole field uncertainties, are estimated to be well below $10 \%$. The measured beam size can be expressed as $\sigma_{M}^{2}=\sigma^{2}+\sigma_{R}^{2}$, where $\sigma$ is the real beam size and $\sigma_{R}$ the monitor resolution. In the approximation of a constant $\beta$ function at the measurement location during the emittance scan, the measured and real beam sizes can be expressed as $\sigma_{M}^{2}=\left(\varepsilon_{n, M} / \gamma\right) \beta$ and $\sigma^{2}=\left(\varepsilon_{n} / \gamma\right) \beta$, where $\varepsilon_{n, M}$ is the measured and $\varepsilon_{n}$ the real emittance. From these expressions:

$$
\varepsilon_{n, M}=\varepsilon_{n}+\sigma_{R}^{2} \gamma / \beta=\varepsilon_{n}+\varepsilon_{n, R} .
$$

The most effective way to reduce the emittance resolution $\varepsilon_{n, R}$ is to improve the profile monitor resolution $\sigma_{R}$.
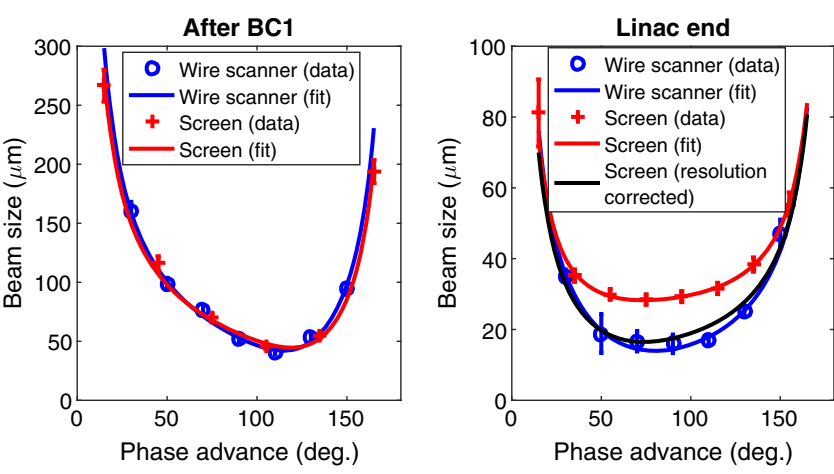

FIG. 4. Horizontal beam sizes after BC1 (left) and at the linac end (right) measured with a wire scanner and a screen. We show the beam-size measurement (data), from which the emittance and optics are calculated, and the reconstructed beam sizes (fit) obtained from the emittance and optics. At the linac end we also show the fitted beam sizes from the screen after subtracting its calculated resolution.

We developed screens with an expected resolution between 10 and $20 \mu \mathrm{m}$ [28] to measure the projected and slice emittance, and wire scanners with $1 \mu \mathrm{m}$ resolution [27] for the projected measurements. Figure 4 shows a measurement of the horizontal projected emittance using the screen and the wire scanner for a beam charge of $10 \mathrm{pC}$ with the same conditions after $\mathrm{BC} 1$ and at the end of the linac. Equivalent results have been obtained in the vertical direction and for a beam charge of $200 \mathrm{pC}$ (not shown). For the measurements after $\mathrm{BC} 1$, at a beam energy of $300 \mathrm{MeV}$, both profile monitors measure equivalent beam sizes and normalized emittances: the emittance measured with the wire scanner is $85 \pm 3 \mathrm{~nm}$, with the screen we get $80 \pm 4 \mathrm{~nm}$. The good agreement between the results obtained with the two monitors corroborates our confidence in the measurements. At the linac end, where the beam energy is $5.5 \mathrm{GeV}$ and the beam size much smaller, the beam sizes determined with the wire scanner are significantly smaller than those measured with the screen. As a consequence, the emittance reconstructed from the wire $(178 \pm 17 \mathrm{~nm})$ is about a factor of 2 smaller than the one determined with the screen $(339 \pm 19 \mathrm{~nm})$. Assuming that the wire scanner is measuring the real beam size, the screen resolution at the linac end is found to be $23 \pm 4 \mu \mathrm{m}$. Figure 4 illustrates how, by construction, the beam sizes measured with the wire scanner are equivalent to the values obtained with the screen after correcting for its estimated resolution. A profile resolution of $23 \mu \mathrm{m}$ is equivalent to an emittance resolution of $98 \mathrm{~nm}$ for a beam energy of $5.5 \mathrm{GeV}$, while the $1 \mu \mathrm{m}$ resolution of the wire scanner corresponds to an emittance resolution of only $0.2 \mathrm{~nm}$ (based on the average $\beta$ function at the profile monitor during the measurement, which is $58 \mathrm{~m}$ ). These results indicate that we have to employ wire scanners for the projected measurements performed at the end of the machine but that they are not necessary at $\mathrm{BC} 1$. 


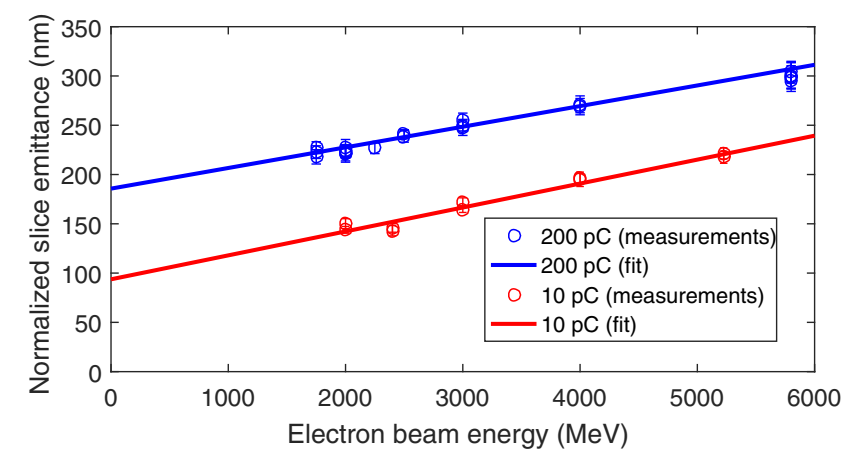

FIG. 5. Measured normalized slice emittance as a function of beam energy for beam charges of 200 and $10 \mathrm{pC}$.

According to Eq. (1), there are two other ways to improve the emittance resolution. First, the measurement optics should be optimized to have a large $\beta$ function at the profile monitor. In our case, for example, the $\beta$ function for the slice emittance measurement at the linac end is between 60 and $80 \mathrm{~m}$. This approach is limited by lattice constraints and by the requirement to scan the phase advance during the emittance measurement, which can be challenging for larger $\beta$ functions. Another possibility to improve the emittance resolution is to reduce the beam energy at the location where the measurement takes place. This approach also has its limitations: the measurement cannot be done at arbitrarily low energies, since below a certain critical energy space-charge effects would deteriorate the beam quality [29].

Equation (1) further suggests the possibility to extract the real emittance $\varepsilon_{n}$ and the profile resolution from the linear dependence of the measured emittance on the electron beam energy (in a similar vein the dependence on the $\beta$ function could be exploited). This novel and simple method works if the $\beta$ function is constant during the emittance scan and if space-charge forces or other collective effects do not affect the normalized emittance over the energy range in which the emittance measurements are done. Figure 5 shows slice emittance values measured at the linac end as a function of the beam energy with corresponding linear fits for beam charges of 200 and $10 \mathrm{pC}$. The measurements were done after beam optimization and for energies down to around $2 \mathrm{GeV}$, where space-charge effects are negligible at our peak currents. The $\beta$ function is rather constant with an average value of $70 \mathrm{~m}$. The data shown in Fig. 5 clearly exhibit the expected linear behavior. For $200 \mathrm{pC}$, the fit yields a normalized emittance of $186 \pm 3 \mathrm{~nm}$ and a profile resolution of $27 \pm 7 \mu \mathrm{m}$. For the $10 \mathrm{pC}$ case, we find a normalized emittance of $94 \pm 5 \mathrm{~nm}$ and a profile resolution of $29 \pm 8 \mu \mathrm{m}$. The resolution of the profile monitor matches the previously found value of $23 \pm 4 \mu \mathrm{m}$. Figure 6 shows an example slice emittance measurement for the case of $200 \mathrm{pC}$ charge and $2 \mathrm{GeV}$ beam energy.

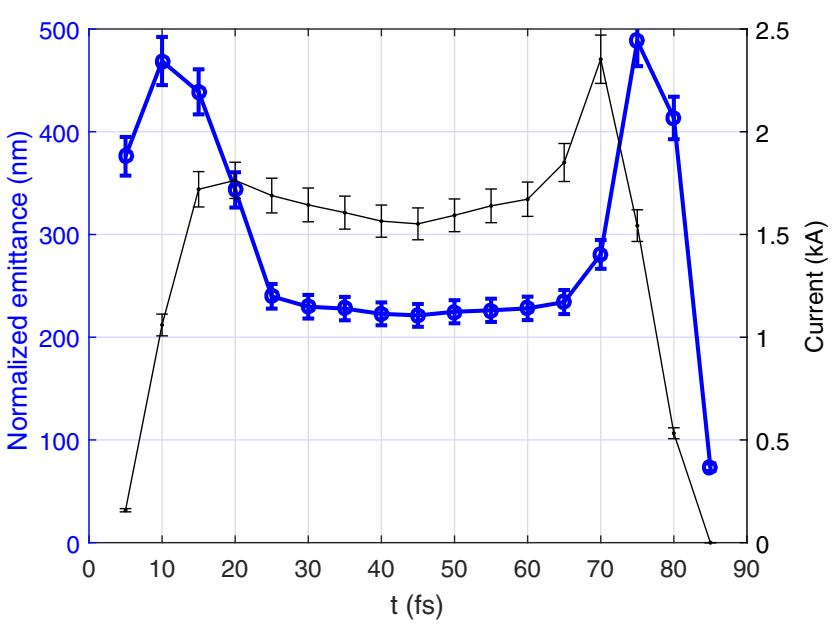

FIG. 6. Slice emittance measurement after nominal compression for a beam charge of $200 \mathrm{pC}$.

Table I presents the slice and projected emittance values obtained after optimization at the different measurement locations for beam charges of 200 and $10 \mathrm{pC}$. The short-term stability of our emittance values is excellent, with reproducibility within a few percent over five consecutive measurements. After each machine setup and reoptimization, the obtained emittance values are mostly reproducible, but may vary up to $10-20 \%$ for the measurements after compression. For $200 \mathrm{pC}$ and after nominal compression, the slice emittance is around $200 \mathrm{~nm}$ and the projected emittances are around 400 and $300 \mathrm{~nm}$ in the horizontal and vertical planes, respectively. For $10 \mathrm{pC}$, the reconstructed final slice emittance is around $100 \mathrm{~nm}$, the projected horizontal emittance is below $200 \mathrm{~nm}$, and the projected vertical emittance is less than $100 \mathrm{~nm}$. The measured emittances for uncompressed bunches are similar to the ones obtained in

TABLE I. Measured emittances for 200 and $10 \mathrm{pC}$ at different locations along the linac. The rms pulse duration $\left(\sigma_{t}\right)$ and core peak current $(I)$ for each case are indicated. Numbers in brackets indicate optimized simulation values [21] (these values correspond to a flat-top longitudinal gun laser profile and refer to the rms emittances).

\begin{tabular}{|c|c|c|c|c|}
\hline $200 \mathrm{pC}$ & \multicolumn{3}{|c|}{ UncompressedAfter BC1 } & Linac end \\
\hline$\sigma_{t}(\mathrm{ps})$ & $\approx 3$ & & \multicolumn{2}{|c|}{$\approx 0.4-0.5 \approx 0.03-0.04$} \\
\hline$I(\mathrm{~A})$ & $\approx 20$ & & \multicolumn{2}{|c|}{$\approx 120-150 \approx 1500-2000$} \\
\hline Slice $(\mathrm{nm})$ & $\approx 150$ & $(140)$ & $\approx 200$ & $\approx 200$ \\
\hline projected horizontal $(\mathrm{nm})$ & $\approx 200$ & $(210)$ & $\approx 250$ & $\approx 400$ \\
\hline projected vertical (nm) & $\approx 200$ & $(210)$ & $\approx 250$ & $\approx 300$ \\
\hline $10 \mathrm{pC}$ & \multicolumn{3}{|c|}{ UncompressedAfter BC1 } & Linac end \\
\hline$\sigma_{t}(\mathrm{ps})$ & $\approx 1.4$ & & $\approx 0.2$ & $\approx 0.003$ \\
\hline$I(\mathrm{~A})$ & $\approx 2.5$ & & $\approx 18$ & $\approx 1200$ \\
\hline Slice & $\approx 60$ & (40) & $\approx 80$ & $\approx 100$ \\
\hline projected horizontal (nm) & $\approx 90$ & & $\approx 90$ & $\approx 180$ \\
\hline projected vertical (nm) & $\approx 90$ & & $\approx 90$ & $\approx 90$ \\
\hline
\end{tabular}


numerical optimizations [21], especially for the standard $200 \mathrm{pC}$ case, in which we put more effort to minimize the emittance.

Compression increases the slice emittance by about one third for $200 \mathrm{pC}$ and by one half for $10 \mathrm{pC}$, compared to the uncompressed beam. We attribute this to three-dimensional CSR effects imposed during bunch compression [30]. The final vertical projected emittance is preserved for $10 \mathrm{pC}$ and increases by $50 \%$ for $200 \mathrm{pC}$. This deterioration mostly stems from spurious wakefield effects, which are more significant at higher charges. The final horizontal projected emittance is about twice the initial emittance. In the $200 \mathrm{pC}$ case, we relate this increase to wakefields and compression effects, whereas at $10 \mathrm{pC}$ it is likely due to compression effects, in particular to strong beam tilts difficult to compensate fully with the available magnetic strength of our quadrupole and sextupole correctors.

In conclusion, we have obtained slice emittances as low as $200 \mathrm{~nm}(100 \mathrm{~nm})$ for a beam charge of $200 \mathrm{pC}(10 \mathrm{pC})$ with peak currents at the $\mathrm{kA}$ level. These values represent new benchmarks for electron linear accelerators. We attribute our low emittance values to the excellent quality of our hardware, the machine design, the careful optimization procedure, and the possibility to reconstruct very small emittances with a robust, precise, and high-resolution measurement procedure. At a bunch charge of $200 \mathrm{pC}$, these low-emittance beams have produced FEL radiation with a wavelength of $0.1 \mathrm{~nm}$ and pulse energies above $0.5 \mathrm{~mJ}$ [31], much higher than the $0.15 \mathrm{~mJ}$ foreseen in the SwissFEL CDR [10]. Most of the improvement can be traced back to the fact that the real emittances are a factor of two better than the initially assumed $430 \mathrm{~nm}$. For $10 \mathrm{pC}$, our beams have already produced high-power FEL radiation with pulse durations of a few fs and shorter [32].

Our results demonstrate the feasibility of generating x-ray FEL radiation with much lower-energy electron beams than presently operating FEL facilities. Taking the example shown in Fig. 1, the same performance obtained with the SwissFEL CDR parameters $(5.8 \mathrm{GeV}$, $430 \mathrm{~nm})$ can be achieved with our measured emittance values $(200 \mathrm{~nm})$ and a beam energy of less than $4.5 \mathrm{GeV}$. Therefore, our emittance values pave the way for even more compact and affordable FEL facilities, such as the CompactLight project [33], potentially multiplying the number of x-ray FELs worldwide and hence facilitating access to these scientifically prolific machines.

We thank all the technical groups involved in the operation of SwissFEL, in particular the laser, the diagnostics, the rf, and the operation teams. Special thanks go to Nicole Hiller for calibrating the screens employed in the emittance measurements, to Alexandre Gobbo for writing the software to acquire the wire-scanner data, and to Andrej Babic for the development of the scan tool used in the emittance measurements. This work has been supported by the SNF Grant No. 200021175498. *eduard.prat@psi.ch

[1] B. W. J. McNeil and N. R. Thompson, Nat. Photonics 4, 814 (2010).

[2] C. Pellegrini, A. Marinelli, and S. Reiche, Rev. Mod. Phys. 88, 015006 (2016).

[3] P. Emma et al., Nat. Photonics 4, 641 (2010).

[4] T. Ishikawa et al., Nat. Photonics 6, 540 (2012).

[5] H.-S. Kang et al., Nat. Photonics 11, 708 (2017).

[6] H. Weise and W. Decking, in Proceedings of the 38th International Free-Electron Laser Conference, Santa Fe, NM, USA, 2017 (JACoW, Geneva, 2018), p. 9.

[7] C. Milne et al., Appl. Sci. 7, 720 (2017).

[8] E. L. Saldin, E. A. Schneidmiller, and M. V. Yurkov, Opt. Commun. 281, 1179 (2008).

[9] M. Xie, in Proceedings of the 1995 Particle Accelerator Conference, Dallas, Texas, USA, 1995 (IEEE, Piscataway, 1996), p. 183

[10] R. Ganter, SwissFEL Conceptual Design Report, PSI Report No. 10-04, 2012.

[11] D. H. Dowell, P. R. Bolton, J. E. Clendenin, P. Emma, S. M. Gierman, W. S. Graves, C. G. Limborg, B. F. Murphy, and J. F. Schmerge, Nucl. Instrum. Methods Phys. Res., Sect. A 507, 327 (2003).

[12] Y. Ding et al., Phys. Rev. Lett. 102, 254801 (2009).

[13] F. Zhou, A. Brachmann, P. Emma, S. Gilevich, and Z. Huang, Phys. Rev. Accel. Beams 15, 090701 (2012).

[14] M. Krasilnikov et al., Phys. Rev. Accel. Beams 15, 100701 (2012)

[15] E. Prat, M. Aiba, S. Bettoni, B. Beutner, S. Reiche, and T. Schietinger, Phys. Rev. Accel. Beams 17, 104401 (2014).

[16] H.-S. Kang et al., J. Synchrotron Radiat. 26, 1127 (2019).

[17] T. Schietinger et al., Phys. Rev. Accel. Beams 19, 100702 (2016).

[18] J. S. Fraser, R. L. Sheffield, E. R. Gray, and G. W. Rodenz, IEEE Trans. Nucl. Sci. 32, 1791 (1985); for a more recent review see, e.g., C. Hernandez-Garcia, P. G. O'Shea, and M. L. Stutzman, Phys. Today No. 2 61, 44 (2008).

[19] S. Bettoni, M. Pedrozzi, S. Reiche, and T. Schietinger, in Proceedings of the 2nd International Particle Accelerator Conference, San Sebastian, Spain (JACoW, Geneva, 2011), p. 3107.

[20] P. Craievich et al., in Proceedings of the 35th International Free-Electron Laser Conference, New York, USA (JACoW, Geneva, 2013), p. 236.

[21] S. Bettoni, M. Pedrozzi, and S. Reiche, Phys. Rev. ST Accel. Beams 18, 123403 (2015).

[22] E. Prat and M. Aiba, Phys. Rev. ST Accel. Beams 17, 052801 (2014).

[23] B. E. Carlsten and T. O. Raubenheimer, Phys. Rev. E 51, 1453 (1995).

[24] E. L. Saldin, E. A. Schneidmiller, and M. V. Yurkov, Nucl. Instrum. Methods Phys. Res., Sect. A 398, 373 (1997).

[25] M. W. Guetg, B. Beutner, E. Prat, and S. Reiche, Phys. Rev. ST Accel. Beams 18, 030701 (2015).

[26] E. Prat, Nucl. Instrum. Methods Phys. Res., Sect. A 743, 103 (2014). 
[27] G. L. Orlandi, P. Heimgartner, R. Ischebeck, C. Ozkan Loch, S. Trovati, P. Valitutti, V. Schlott, M. Ferianis, and G. Penco, Phys. Rev. Accel. Beams 19, 092802 (2016).

[28] R. Ischebeck, E. Prat, V. Thominet, and C. O. Loch, Phys. Rev. ST Accel. Beams 18, 082802 (2015).

[29] M. Ferrario, M. Migliorati, and L. Palumbo, Space Charge Effects, Contribution to the CERN Accelerator School:
Advanced Accelerator Physics Course, Trondheim, Norway (CERN, Geneva, 2013).

[30] S. Bettoni, M. Aiba, B. Beutner, M. Pedrozzi, E. Prat, S. Reiche, and T. Schietinger, Phys. Rev. Accel. Beams 19, 034402 (2016).

[31] E. Prat et al. (to be published).

[32] A. Malyzhenkov et al. (to be published).

[33] CompactLight Project Homepage, http://www.compactlight .eu. 